\title{
DYNAMIC DEFORMATIONS IN COORDINATE MEASURING ARMS USING VIRTUAL SIMULATION
}

\author{
Cuesta, E.; Mantaras, D. A.; Luque, P.; Alvarez, B. J. \& Muina, D. \\ Dept. of Construction \& Manufacturing Engineering, University of Oviedo, 33204 Gijon, Spain \\ E-Mail: ecuesta@uniovi.es
}

\begin{abstract}
This paper presents a study for assessing the dynamic structural deformations of one of the most modern, and portable, measurement equipment: the Coordinate Measuring Arms (CMAs or AACMMs). The study of the measurement errors derived from the use of these instruments is still controversial due to a certain lack of traceability and reliability in measurements due to the influence of the human factor acting on a complex 3D structure (with 6 or 7 degrees-of-freedom). When contact measurements are taken into account, the human factor variable originates non-uniform contact forces, lack of stability, different velocities and accelerations, among unpredictable probing trajectories. All these factors lead to conclude that in every manual measurement involving a CMA there is a structural dynamic deformation caused by the approach movement before probing a contact point as well as by the force exerted during probing. In order to determine the amplitude of this deformation and its distribution along the CMA structure a new methodology has been developed. Both depend on the followed trajectory and on the probing force amplitude and orientation. This work presents the methodology and steps required for the construction of the virtual simulation model. The method employs 3D modelling of all elements of the CMA structure, Finite Element Analysis (FEA) software and multibody simulation techniques with flexible bodies. This set of software tools is nowadays capable of creating dynamic virtual models for the dynamic analysis of complex mechanisms (machines, vehicles...) in optimization tasks. The application of these tools supposes a novel approach to the study of the CMA metrological behaviour.

(Received in December 2014, accepted in May 2015. This paper was with the authors 1 month for 1 revision.)
\end{abstract}

Key Words: Coordinate Measuring Arms, Dynamic Deformations, Dynamic Simulation

\section{INTRODUCTION}

Nowadays production companies employ many resources, time and costs in the inspection of final parts, but also in inspection of intermediate parts, fixturing systems, machine tools, gauges, etc. Many of these auxiliary parts are also needed to manufacture the final parts. In this scope, the optimization of the on-line and on-site inspection tasks can arise as a significant technological and commercial advantage. Portable coordinate measuring arms (AACMMs or CMAs) are ideal for inspections "on site" due to their flexibility (for GD\&T inspection) and their lower cost compared to fixed CMMs. Certainly the use of CMAs is producing very significant time and cost savings. However this type of equipment has difficulties to ensure traceability for several reasons. They are designed to measure in noncontrolled temperature environments and, moreover, the operation is completely manual, therefore contact forces are variable, point sampling is not uniform and not repeatable, stability is low, velocities and accelerations are different from point to point, etc. In this sense there are many studies that suggest that variations in the positions of the CMA components (and of the encoders) have great relevance in the precision and repeatability of the measurement. The variability in trajectories of the CMA components, along with the variability in the contact forces, lead to a scenario that could invalidate the use of CMA on parts where the size, the accessibility or the distance between features involve measuring with very different CMA orientations. 
Existing studies on accuracy in CMA contact measurement are focused on its verification and calibration by analysing its particular structure consisting of rigid segments linked by joints equipped with angular encoders [1-3]. For example, Santolaria et al. [4] optimized the parameters of the CMA kinematic model to improve the accuracy in the measurement of an artefact made with spheres. This optimization includes all sources of error on the kinematic model. In a later work [5], once the CMA has been calibrated, Santolaria et al. added a correction for adjusting the parameters according to the temperature variations. More recently, Ostrowska et al. [6] determine the measurement uncertainty of a CMA by means of a virtual kinematic model using statistical methods (Monte Carlo simulation). Recent research also suggests that, apart from the contributions of the CMA structure, the influence of the operator should be taken into account when assessing uncertainty [7-10], mainly due to the lack of repeatability in point sampling, stability and forces, or lack of reproducibility for different operators.

In the area in which this paper focuses, the errors induced by the structure of the CMA (position of its components), the more interesting analysis has been made by Vrhovec and Munih [11]. Their approach studies deflection of the longer arm segments using optical sensors. The deviation of each segment is evaluated and introduced into the kinematic model of CMA, but this method is expensive (requires the use of laser interferometer) and difficult to implement for the simultaneous reading of the deflection in all segments. However, Vrhovec et al. [12] suggest that the error due to the deflection caused by contact forces is one of the most influential and that it can be compensated or at least taken into account for calculating measurement uncertainty. Preliminary studies from this research team have proved that the forces exerted during measurements errors can produce errors up to hundreds of micrometres [13-15]. In any case, the contact force and the deflection on the structure are parameters not currently considered in the internal mathematical calculations of the CMA software.

Moreover, the existing standards ASME B89.4.22-2004 [16], VDI / VDE 2617 [17] and the recent ISO 10360-12 (currently under development) [18] are aimed only at verification for acceptance or recertification of CMAs, but do not provide specific guidelines about how to structurally improve its accuracy, avoid certain movement trajectories, control probing forces, etc. Furthermore, these standard describe verification or calibration procedures, where a special probe is used (steel sphere of $15 \mathrm{~mm}$ in diameter, for example, or a completely new design [19]), that involve the measurement of ball-bars or similar artefacts, including features quite different to the usual geometries found in a mechanical part. At the industrial level, in case the CMA needs to be adjusted or corrected, it is sent to the manufacturer that proceeds to calibrate it in a blind process to the final user.

In order to study the deformations of the entire structure simultaneously, both statically and dynamically, a tool was developed for evaluating the elastic deformations generated at each body of the CMA, checking how they affect the measurement results. Undoubtedly, this tool could prevent incorrect, extreme or careless handling and justify why extreme positions of the encoders induce large systematic errors in the measurement.

Moreover, quantification of the deformation induced by the movement of the structure during any path is an achievement in itself. It has to be taken into account that deformations are always present as the CMA bodies are not perfectly rigid.

The application presented here starts from the previous experience of a basic study of measuring force $[13,14]$ as abstracted in Fig. 1. This research was performed using a strain gauges arrangement (Fig. 1, left) mounted directly on the rigid probe -hard probe- near the last joint of an available CMA (ROMER $($ C model). Within this previous research, a customized application was developed to read and record the signals (Fig. 1, right) of the probing force components in real-time. The virtual simulation model is now developed from 
the data of the probing force provided by the strain gauges arrangement as well as the signals provided by the CMA angular encoders. Subsequently, virtual simulations are carried out for a given trajectory of the CMA where its structural deformations can be evaluated in any situation. In particular, the deformations are evaluated for a trajectory from a resting position to a relative static position between the workpiece and the hard probe (approach movement) or for the instant when the probe is touching the part and a variable probing force is applied (probing movement). Moreover, this analysis of the CMA deformations can be also performed dynamically.

\section{METHODOLOGY FOR VIRTUAL CMA ANALYSIS}

A methodology for evaluating the deformation of CMA bodies during measurement operations is proposed. This methodology is based on computer simulation of the measurement process, and a virtual experimental program is put forward. Since the measuring operation using a CMA is a dynamic process, that involves movements and efforts performed by an operator, the most suitable simulation tools are those that allow the characterization of complex multibody systems.

Typically, for simplicity and computational efficiency, this type of models considers bodies as rigid (non-deformable). In this case, as the influence of the deformation on different elements of CMA will be evaluated, a three-dimensional multi-body model was developed taking into account the elasticity of the different bodies.

The MSC-ADAMS ${ }^{\circledR}$ software was used for the implementation the model of the virtual CMA and measurement process. This software simulates dynamic models with flexible bodies in a shorter time than other explicit FEA software packages do. This type of software is successfully used in different fields, such as in the automotive sector [20], or in different manufacturing processes $[21,22]$, etc., for the same purpose of this work. Utilizing multibody dynamics solution technology, MSC-ADAMS runs nonlinear dynamics in a fraction of the time required by FEA solutions. Loads and forces computed by MSC-ADAMS simulations improve the accuracy of FEA by providing better assessment of how they vary throughout a full range of motion and operating environments.

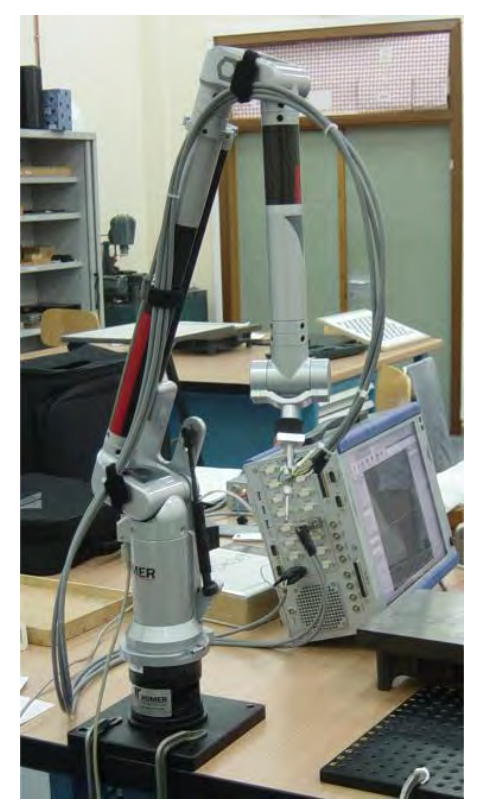

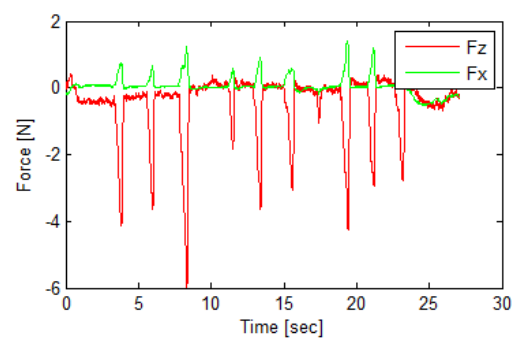

Operator A

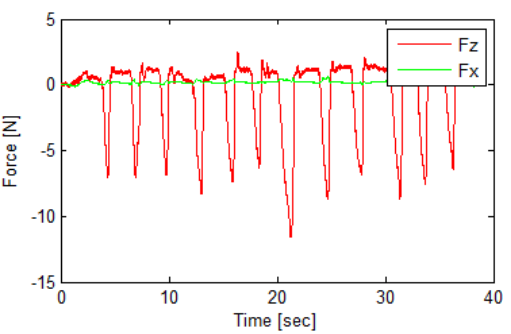

Operator C

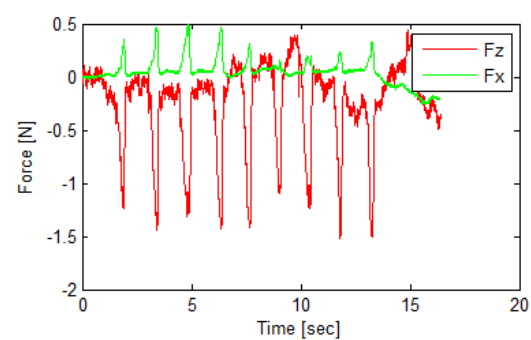

Operator B

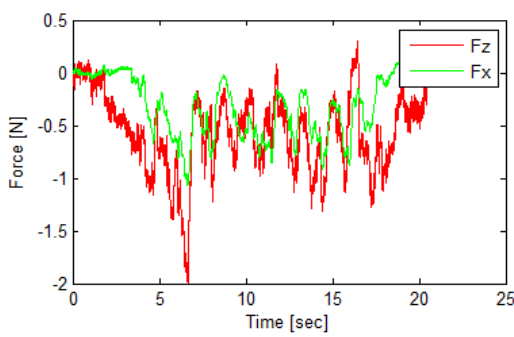

Operator D

Figure 1: Left - Available CMA with the gauge arrangement over the rigid probe; Right - Four examples of the force signals captured in real-time for several operators. 
The developed virtual model corresponds closely with the available instrument used for the first tests $[13,14]$, which showed that both the type of geometry to measure $[8,9]$ as the human factor [10] had great influence on the quality of measurements. Specifically, the CMA used for the development of the methodology (ROMER Sigma 2048 of Hexagon Metrology(C) has a spherical working range of $\varnothing 1800 \mathrm{~mm}$, length accuracy specification of $\pm 0.025 \mathrm{~mm}$ and uncertainty of $\pm 0.045 \mathrm{~mm}$ given by the manufacturer.

Real-time readings of various components of the probing forces (Fig. 1, right) were monitored for different operators on measurements of planar surfaces and inner cylinders by means of strain gauges assembled on the CMA hard probe [13, 14]. Some of the values and directions of the monitored forces will be subsequently introduced to the virtual model in order to use them as valid "seeds" for the dynamic structural deformations survey.

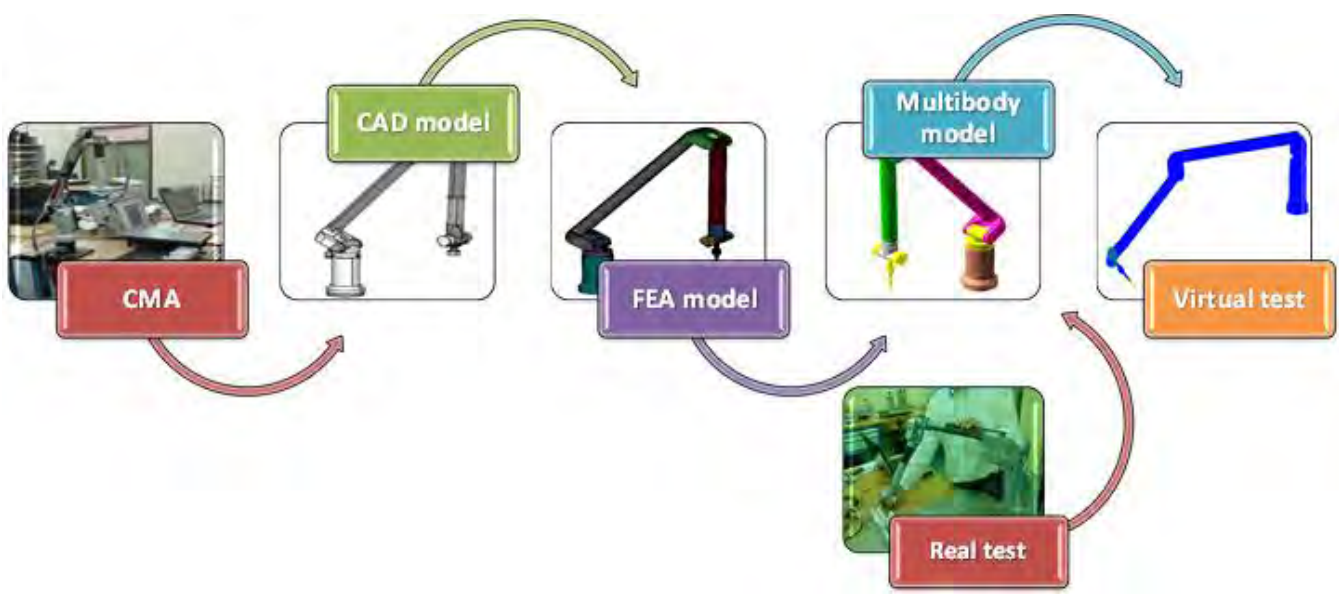

Figure 2: Methodology followed for virtual CMA analysis.

The proposed methodology is developed sequentially in the following steps (Fig. 2):

- Development of a full 3D CAD model of the CMA;

- Meshing and extraction of stiffness matrices using finite element analysis tools (FEA model);

- Implementation of a multi-body model in MSC-ADAMS software;

- Performing real tests of a measurement process (Real test);

- Simulation of real tests by means of virtual measurement tests;

- Analysis of results and conclusions.

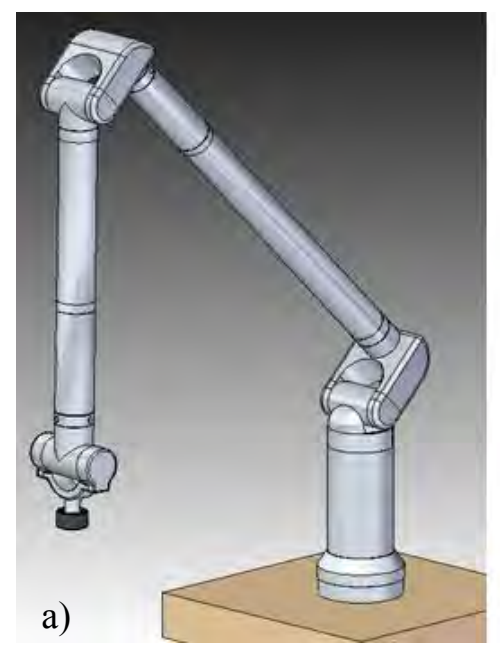

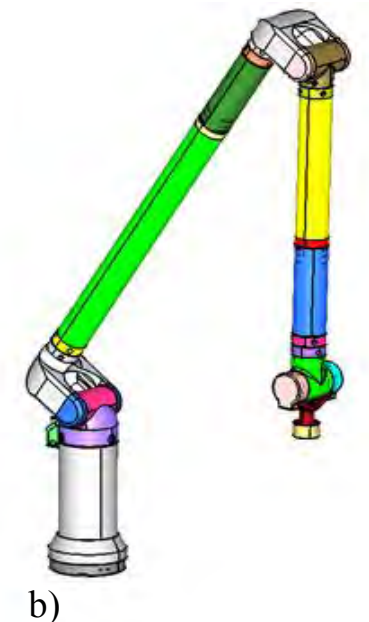

b)

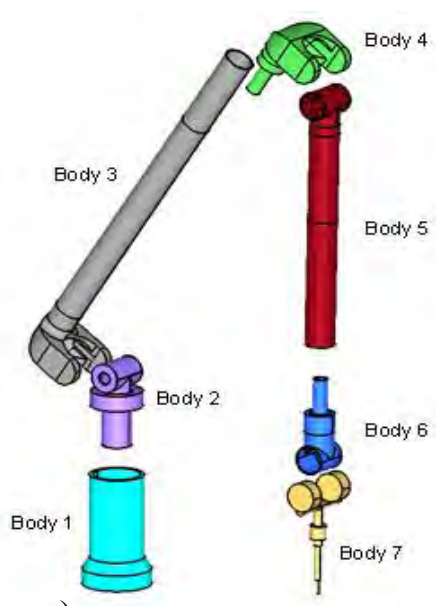

c)

Figure 3: a) Full CAD model of the CMA, b) the seven bodies with complex geometries, c) simplified bodies separately shown (exploded view). 


\subsection{CMA virtual model}

A three-dimensional multibody model of the CMA was developed taking into account the flexibility of each component. To do this, a detailed CAD model of the CMA, with complex body geometries, bearings, lots, bolts, joints, etc., was implemented.

Given the stiffness of the bolted joints, they are not taken into account, so that contacts between joined parts were not modelled and the bolted bodies were considered as one body. The first step consists of dividing the full CAD model into seven different bodies connected by kinematic joints. In the second step the complex geometries of each body were simplified (Fig. $3 \mathrm{~b}$ and Fig. 4), by removing unnecessary features (small surface fillets, holes, key seats, countersinks, etc.) in order to improve the mesh quality and decrease the computation times. Next, the complex bodies were partitioned into groups of smaller connected solids to make them mappable (Fig. 5), in order to put forward a meshing process. The meshed bodies will be introduced in Finite Element Analysis software.

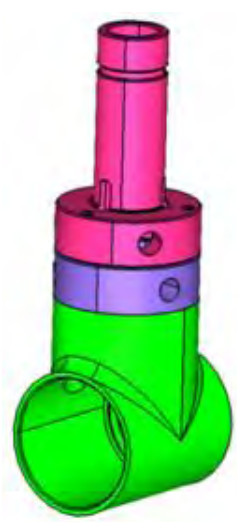

Figure 4: Customized CAD for body 6.
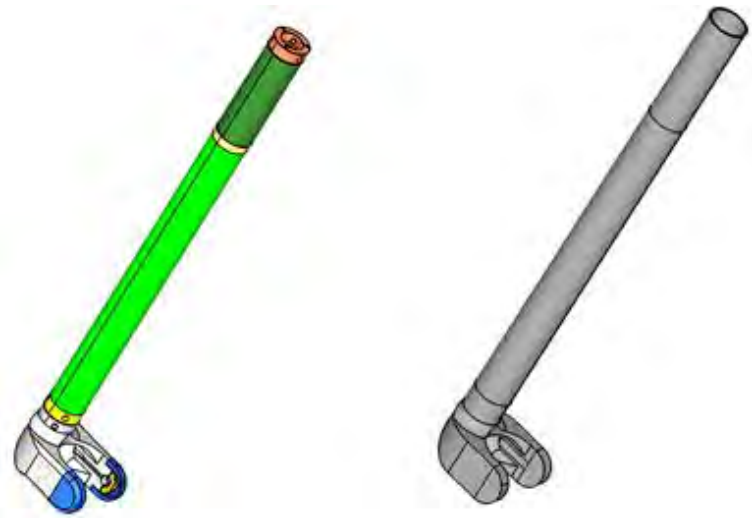

Figure 5: Geometry partitioned of body 3.

In the third step the solids were meshed (Fig. 6) with a pure hexahedral mesh and a higher order 3D 20-node solid element was used. The mesh results are presented in Table I for the seven bodies.

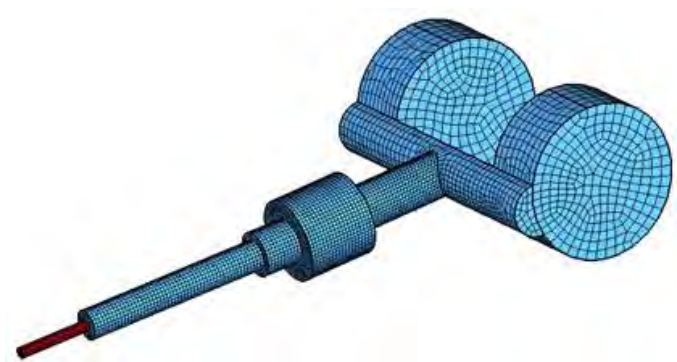

Figure 6: Example of customized mesh (Body 7).

Table I: Mesh parameters for all the bodies.

\begin{tabular}{|c|c|c|}
\hline Body & Elements & Nodes \\
\hline 1 & 3407 & 7718 \\
\hline 2 & 2150 & 4208 \\
\hline 3 & 25780 & 50890 \\
\hline 4 & 2560 & 5373 \\
\hline 5 & 7010 & 13627 \\
\hline 6 & 1580 & 3080 \\
\hline 7 & 4821 & 9072 \\
\hline
\end{tabular}

Once the bodies are meshed, the material properties are defined. In this case, the bodies are made of different materials; the bodies 1,2, 4 and 6 are made of aluminium, the bodies 3 and 5 are made of aluminium and carbon fibre composite and the body 7 is made of four different materials (Table II), carbon fibre composite, aluminium, stainless steel (for the stylus) and ruby (for the probe tip, sphere of $4 \mathrm{~mm}$ diameter). Due to the small size of this part and the high stiffness of its material this tip was not meshed. This part of the body 7 is considered as a rigid body and is implemented in ADAMS as a spherical body connected to an interface point by a rigid joint. This improves the calculation of the three-dimensional contact between the probe and the objects during the multibody simulation of a measure process, improving the accuracy of the simulation results. 
All materials were considered as linear and isotropic due to the nonlinear properties are not included in the modal neutral file. The material properties used can be seen in the Table II. The metallic materials are isotropic but the composite material at representative unit cell level is homogeneous and orthotropic [23]. The composite used in the bodies 3 and 5 is a 3D fabric composite in which the fibrous reinforcements are interlaced in multi-directions. This type of composite have more isotropic response compared with biaxial woven fabrics [24]. Different studies reveal the more isotropic behaviour with small difference in young modulus at any direction [25]. So, for the simulation developed in the present paper, consider the composite as an isotropic material does not affect the quality of results.

Table II: CMA material properties.

\begin{tabular}{|l|c|c|c|c|}
\hline \multicolumn{1}{|c|}{ Material } & Bodies & $\begin{array}{c}\text { Young } \\
\text { moduli } \\
{[\mathrm{MPa}]}\end{array}$ & $\begin{array}{c}\text { Density } \\
{\left[\mathrm{kg} / \mathrm{m}^{3}\right]}\end{array}$ & $\begin{array}{c}\text { Poisson } \\
\text { ratio }\end{array}$ \\
\hline Aluminium & all & 70000 & 2700 & 0.333 \\
\hline Composite & $3 \& 5$ & 115000 & 1500 & 0.22 \\
\hline Stainless steel & 7 & 187500 & 8000 & 0.3 \\
\hline
\end{tabular}

Table III: Torsion and flexion modes.

\begin{tabular}{|c|c|c|}
\hline Body & $\begin{array}{c}\text { First torsion } \\
\text { mode }[\mathrm{Hz}]\end{array}$ & $\begin{array}{c}\text { First flexion } \\
\text { mode }[\mathrm{Hz}]\end{array}$ \\
\hline 1 & 157.3 & 179.6 \\
\hline 2 & 65.6 & 81.5 \\
\hline 3 & 22.4 & 23.7 \\
\hline 4 & 85.1 & 97.7 \\
\hline 5 & 25.7 & 28.8 \\
\hline 6 & 43.8 & 45.2 \\
\hline 7 & 12.3 & 15.4 \\
\hline
\end{tabular}

In the fourth step the interface points, necessary to connect the different bodies in ADAMS, were modelled. An interface point is a node used to apply a joint in ADAMS and must be modelled taking into account that the force of the joint should be applied to the structure by distributing it over an area. In this case, given the geometry of the bodies there were no nodes in their structures suitable for use as an interface points. So nodes, meshed as mass nodes, were created in the geometric centre of each joint for each of the bodies. As can be seen in Fig. 7, in order to distribute the joint force, the interface points were connected to an area of the structure through constraint equations, with zero displacement between the interface point and every node in the selected area.
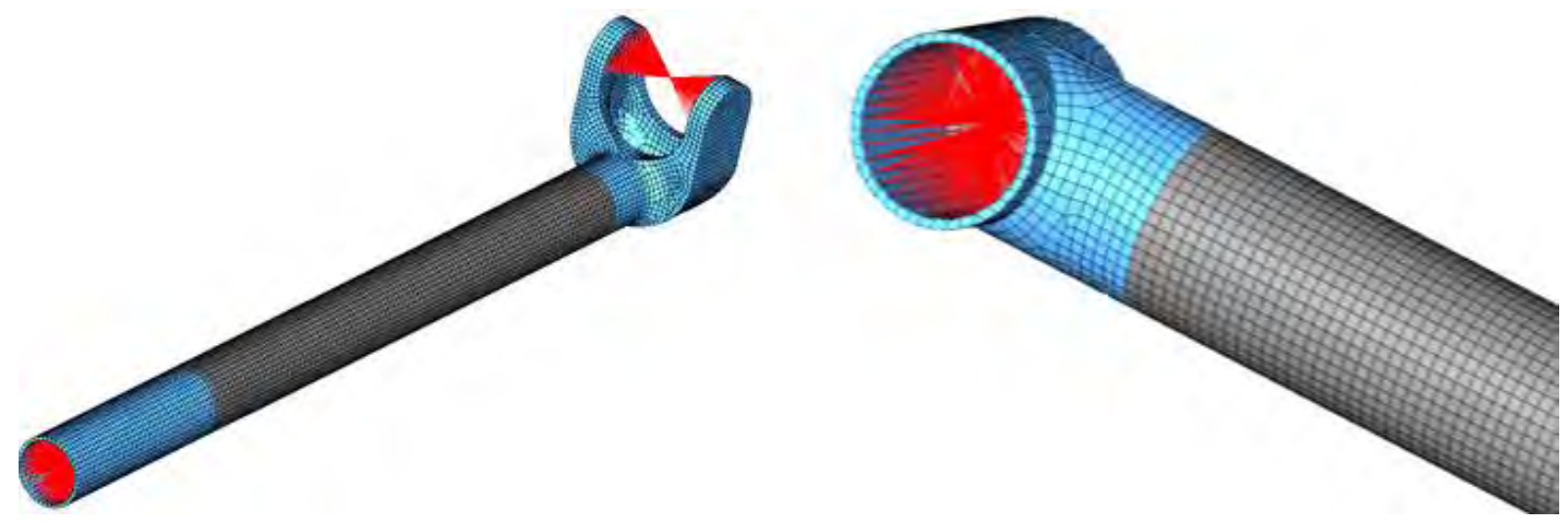

Figure 7: Interface points and constraints of two bodies (3 and 5).

In the fifth step, the modal neutral file (.mnf) of each body was generated. This file contains the flexibility information of the component and includes: geometry, nodal mass and inertia, mode shapes and generalized mass and stiffness for mode shapes. In the table III the first torsion and flexion modes of each body are shown. In the last step, the multibody model was generated in MSC-ADAMS view. Every modal neutral file was imported in MSCADAMS to build the final full device assembly (Fig. 8). To connect the bodies revolute joints, 
with properly orientation, were used, with the exception of the connection between body 1 and ground where a fixed joint was used.

\subsection{Dynamic virtual tests}

It is assumed that the greatest deformation occurs when a measurement is made at extreme positions by moving the device in a continuous movement and a non-slow speed. Therefore, a real measurement test of two separate parallel planes $900 \mathrm{~mm}$ was performed for defining the virtual test. The reason for this choice was to move to positions that limit the ability to work on this type of mechanical arms, working as much as possible far from the idle position.
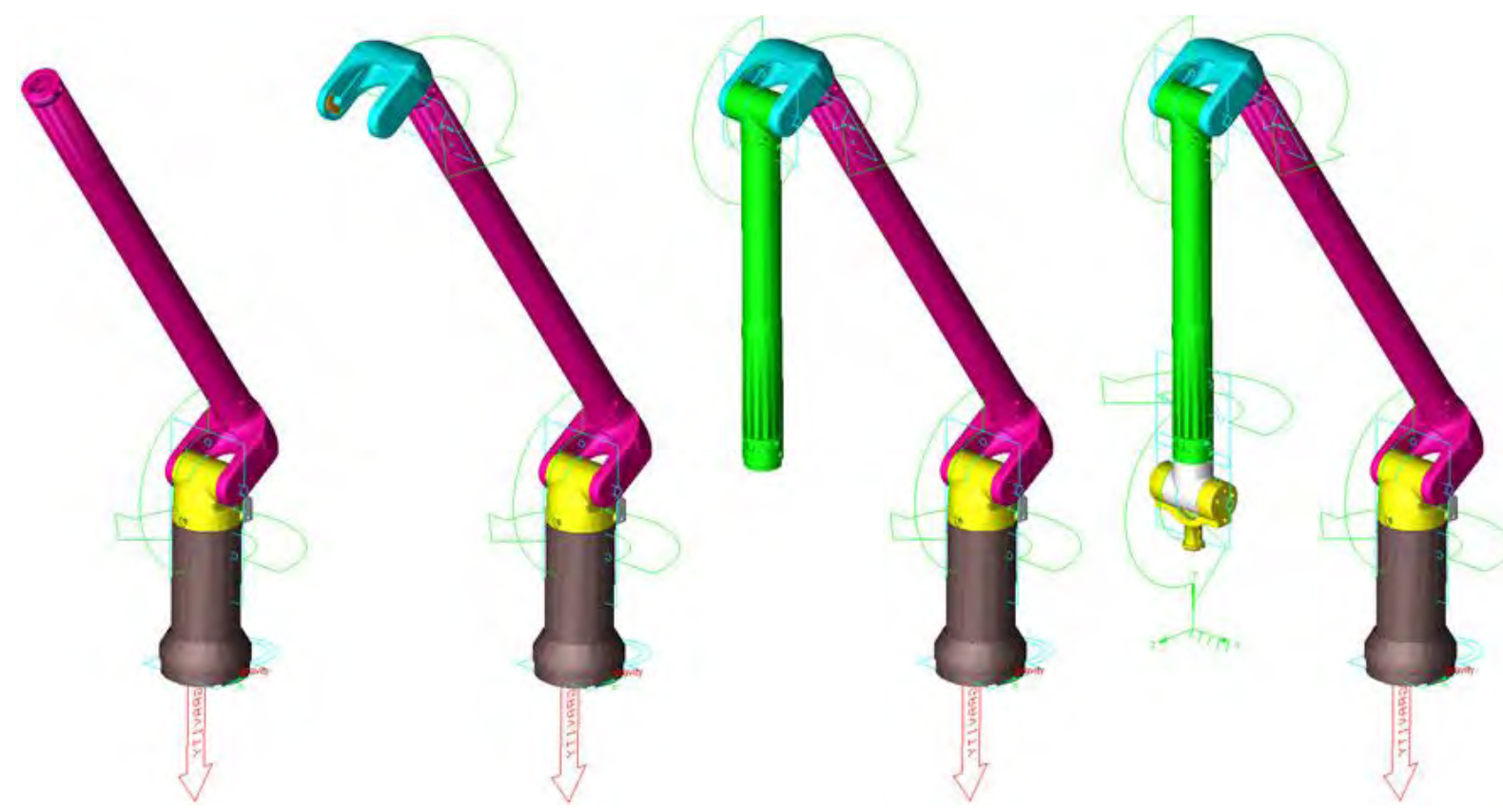

Figure 8: Screen capture of the "under construction" procedure.

During the measurement process performed with the real device the readings from each joint encoder (sensor that measure the angle between bodies of the arms) were recorded. Measures of encoders were used to define the trajectory of the virtual model (angular displacement between each body in time).

In a real measurement process, the operator controls the device movement gripping the probe (body 7) with one hand and with the other hand one of the other arms (bodies 3 or 4). In the measurement test developed, the operator gripped the body 7 and the body 3 in the connection area of the arms. In the simulation software, to define the path of the two points, a first simulation was performed acquiring each joint angle, versus time, registered by the encoders. After this first simulation the trajectory of the two points controlled by the operator is defined. Defined that path in the virtual model a new simulation was performed by recording the deformations and forces of different bodies and joints. In Fig. 9 different frames of the approach movement to the measured object are shown. In this figure, the highlight colour reveals the deformation of each body.

After reaching the position, the measurement is carried out using the probe in its axial direction, so a compression force is applied to this element (similar to $F n$ in Fig. 10). This measurement is repeated later using the probe in perpendicular direction, subjecting the body to a bending stress (Fig. 11). The two measurement processes are simulated using the multibody model but applying a variable force in order to analyse the influence of the force exerted by the operator in the error of the measurement. 


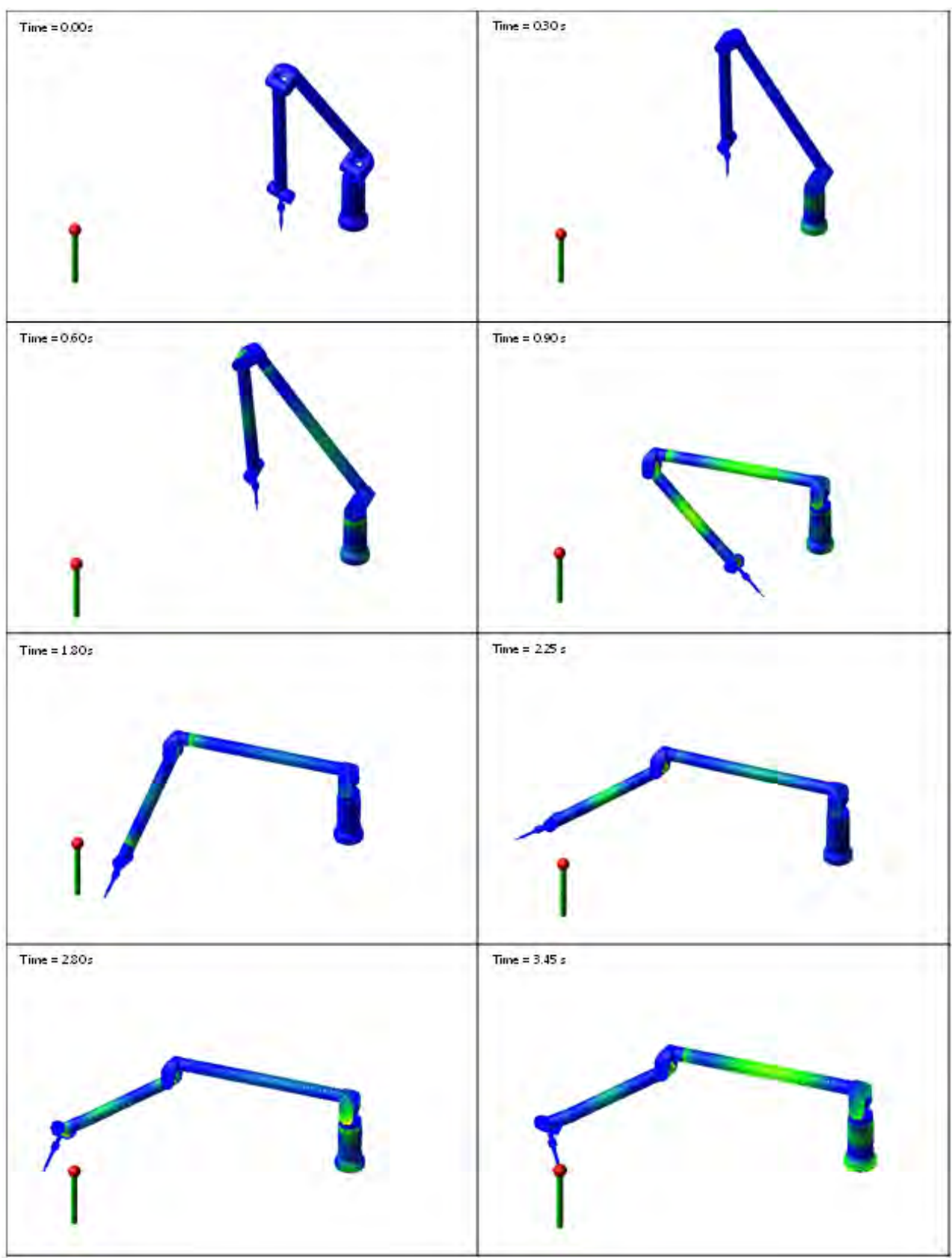

Figure 9: Results obtained with the virtual model of an "approach movement"; the highlight colour indicates structural deformation due to high strengths.
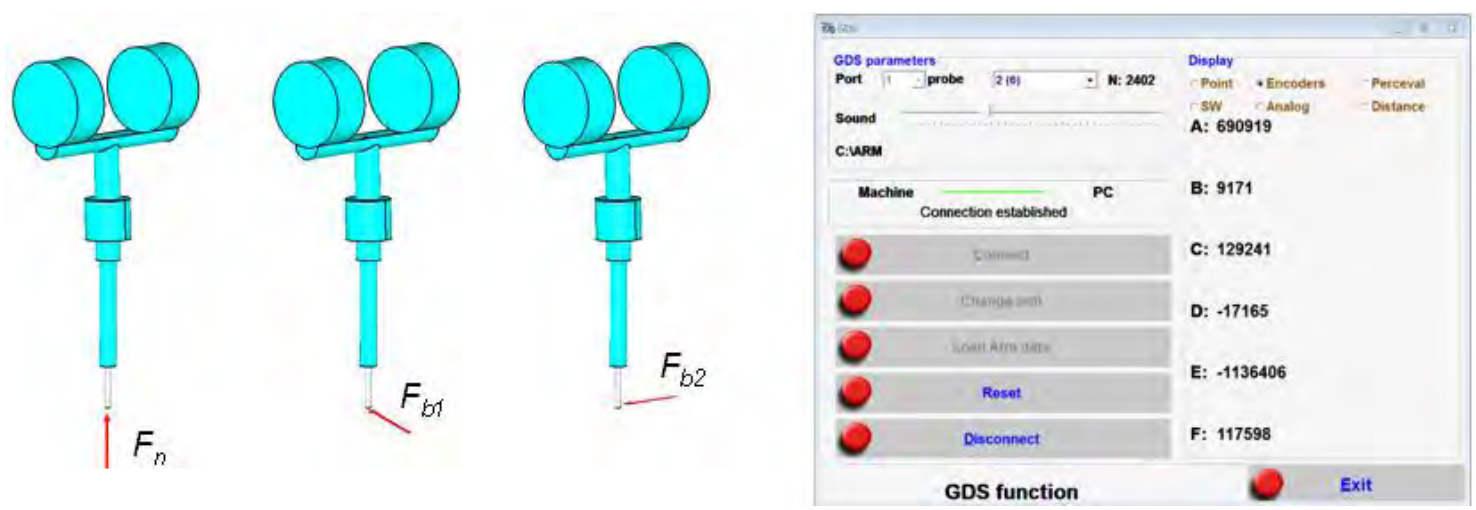

Figure 10: Contact forces over probe in simulated measure process and final values of encoders for such position. 


\section{RESULTS}

The performed simulations show that all bodies deform both during the approach movement towards the workpiece and during probing (probing movement) for measurement. Bodies 3 and 4 were found as the components of the whole CMA that suffered greater deformation in the simulations performed over the virtual model. The deformations during the approach movement to the part depend on the speed and the handling of the CMA. These deformations are smaller than those derived from the probing contact.

It has been shown how the direction of force applied to the probe affects the measurement error. When measurement is carried out in the perpendicular direction to the probe orientation so that no moment is exerted on the joint (Fig. 11), the deformations become smaller and therefore less error is produced. This is due to the specific orientation of the CMA bodies in the analysed measurement orientation, which cannot be extrapolated to other measurement orientations.

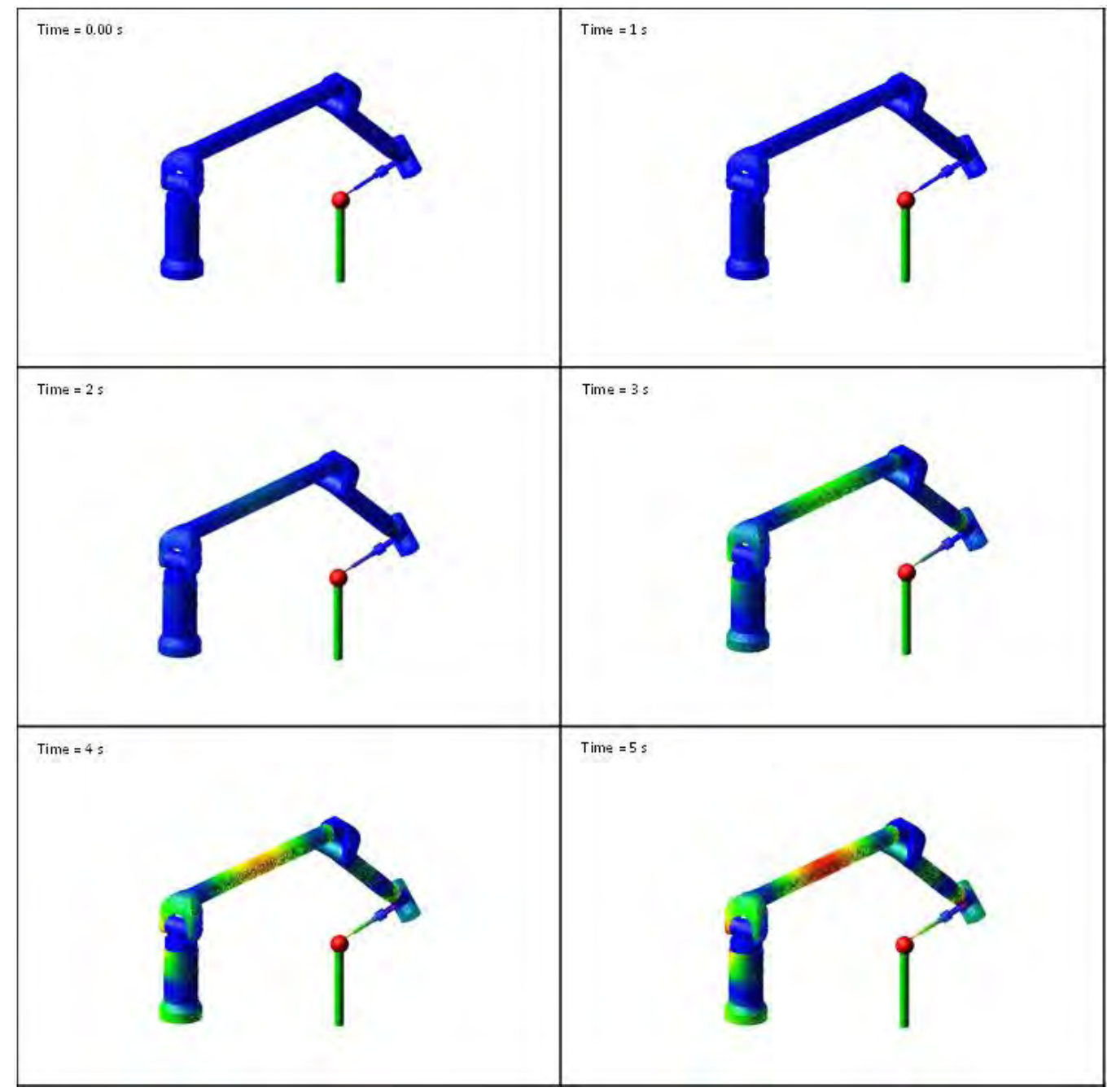

Figure 11: Structural deformations using the probe in its axial direction in a probing movement.

From numerous tests it was found that the magnitude of error increases almost linearly with regard to the contact force applied (Fig. 12), being this error the difference between the measurement result obtained with a structure of non-deformable bodies and the result obtained with the developed model, consisting of flexible bodies.

In the virtual model, actual values were used for probing forces (usually from $1 \mathrm{~N}$ to $5 \mathrm{~N}$ ). Nevertheless extremely high values could be applied for the load on the arm, such as loads up 
to $50 \mathrm{~N}$, difficult to arise in a real situation except for high range CMAs ( $>3200 \mathrm{~mm})$. In any case the maximum values of deformation, and the areas where they occur, can help to optimize the CMA structure and to improve its method of use.

At the probing instant, the deformations that arise due to tensile or compressive loads are negligible, whereas the greatest deformation is produced when a load is applied parallel to the rotation axis (wrist) of the probe $\left(F_{b 2}\right.$ in Fig. 10). This deformation is approximately 6-7 times greater than the obtained when load is applied perpendicularly to the rotation axis $\left(F_{b 1}\right.$ in Fig. $10)$. It could also be checked how the deformation generated in the probe area ultimately affects the deformation of the rest of the structure.

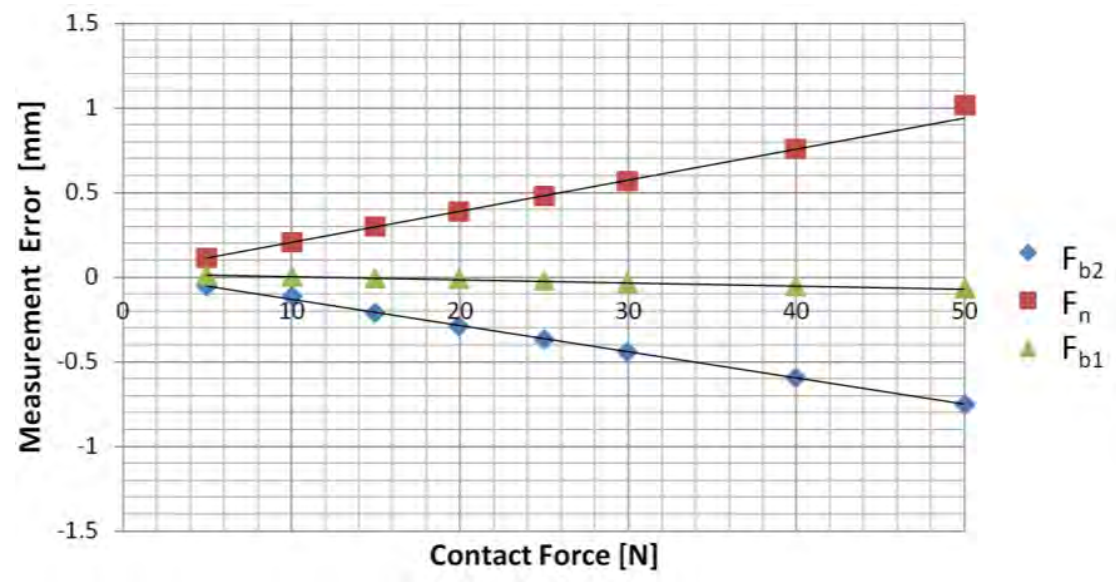

Figure 12: Measurement error versus contact force.

The maximum deformation values obtained from the simulation match the values previously monitored in measurement tests, although the coincidence is not complete as certain dispersion always exists. The force sensor obtains two components, the axial compressive force in the direction of the probe orientation, and the flexion (bending) force, sum of the components in the other transversal directions. On the other hand, the developed virtual model is able to get the three components of the force (compression and two independent flexion forces). Obviously the finite size of the mesh and errors in the definition of the path also contribute to increase the dispersion. Despite these inaccuracies, certainly the model can be considered valid as a tool to simulate dynamically all CMA bodies with the possibility of applying forces of any magnitude and in any direction.

\section{CONCLUSIONS}

It is clear that the developed model can help to quantify how the supporting and probing efforts affect the different mechanisms or segments of the CMA. In summary, the virtual model (multibody with flexible bodies) developed in this work allows for the following analyses:

- Determination, numerically and graphically, of the locations where maximum deformations arise, not only from the static point of view but also dynamically.

- Quantification of efforts over the different bodies of the CMA structure, in order to evaluate the behaviour of these bodies for different measurement tasks, in different working areas, etc.

- Elaboration of models for error compensation that take into account the deformations in the CMA bodies. As a future work, an aid system that prevents extreme postures when using the CMA could be developed. Currently, the measuring system only alert about alignment of CMA bodies or when any join, or its encoder, reaches the limit of angular range. 
- Optimization of the CMA body dimensions, assigning larger thickness for increasing the stiffness of certain zones of the CMA or reducing it on other zones less sensible to deformation.

- Calculation of maximum velocities and accelerations that are suitable for the probing instant, ensuring that the probing is performed not only with the proper force but also with improved stability.

As a final point, a model of this type, aimed at CMAs, has not been designed until now. This might be due to the difficulty of computing, the cost of the software, the enormous difficulties in validating multitasking GD\&T, or the working conditions under non-controlled temperature environments of CMAs. In fact these tools and the methodology employed are oriented to the design and analysis of automotive and aerospace components, where the relevance and benefit that can be obtained from the use of these tools is obvious. On the other hand, addressing this type of study in the metrological scope is nothing usual. By applying this methodology to a manual instrument of high accuracy (CMAs can be considered as portable CMMs) a very interesting point of view contributes to enhance the knowledge of the measurement process. This tool should be essential for the structural optimization of the new generation of CMAs.

\section{ACKNOWLEDGEMENTS}

Authors thank to the Spanish Ministry of Economy and Competitiveness for its financial support of the project "Quality assurance and knowledge modelling applied to portable coordinate measuring systems", ref. DPI2012-36642, through FEDER (ERDF) funds.

\section{REFERENCES}

[1] Kovac, I.; Frank, A. (2001). Testing and calibration of coordinate measuring arms, Precision Engineering, Vol. 25, No. 2, 90-99, doi:10.1016/S0141-6359(00)00057-X

[2] Lin, S.; Wang, P.; Fei, Y.; Chen, C. (2006). Simulation of the errors transfer in an articulationtype coordinate measuring machine, International Journal of Advanced Manufacturing Technology, Vol. 30, No. 9-10, 879-886, doi:10.1007/s00170-005-0122-x

[3] Zhao, S.; Zhu, L.-Q.; Chen, Q.-S.; Pan, Z.-K.; Guo, Y.-K. (2013). A kinematic parameter calibration method for articulated arm coordinate measuring machine, Proceedings of the $6^{\text {th }}$ International Symposium on Precision Mechanical Measurements (ISPMM), Vol. 8916, doi: $10.1117 / 12.2035784$

[4] Santolaria, J.; Aguilar, J.-J.; Yagüe, J.-A.; Pastor, J. (2008). Kinematic parameter estimation technique for calibration and repeatability improvement of articulated arm coordinate measuring machines, Precision Engineering, Vol. 32, No. 4, 251-268, doi:10.1016/j.precisioneng.2007.09. $\underline{002}$

[5] Santolaria, J.; Yagüe, J.-A.; Jiménez, R.; Aguilar, J.-J. (2009). Calibration-based thermal error model for articulated arm coordinate measuring machines, Precision Engineering, Vol. 33, No. 4, 476-485, doi:10.1016/j.precisioneng.2009.01.002

[6] Ostrowska, K.; Gąska, A.; Sładek, J. (2014). Determining the uncertainty of measurement with the use of a Virtual Coordinate Measuring Arm, International Journal of Advanced Manufacturing Technology, Vol. 71, No. 1-4, 529-537, doi:10.1007/s00170-013-5486-8

[7] Ostrowska, K.; Szewczyk, D.; Sladek, J. (2013). Determination of operator's impact on the measurement done using Coordinate Technique, Advances in Science and Technology Research Journal, Vol. 7, No. 20, 11-16, doi:10.5604/20804075.1073045

[8] Cuesta, E.; Alvarez, B. J.; Martinez, S.; Barreiro, J.; González-Madruga, D. (2012). Evaluation of influence parameters on measurement reliability of coordinated measuring arms, AIP Conference Proceedings, Vol. 1413, 214-224, doi: $\underline{10.1063 / 1.4707568}$ 
[9] González-Madruga, D.; Barreiro, J.; Cuesta, E.; González, B.; Martínez-Pellitero, S. (2014). AACMM performance test: Influence of human factor and geometric features, Proceedia Engineering, Vol. 69, 442-448, doi:10.1016/j.proeng.2014.03.010

[10] González-Madruga, D.; Barreiro, J.; Cuesta, E.; Martínez-Pellitero, S. (2014). Influence of human factor in the AACMM performance: a new evaluation methodology, International Journal of Precision Engineering and Manufacturing, Vol. 15, No. 7, 1283-1291, doi:10.1007/ s12541-014-0468-9

[11] Vrhovec, M.; Munih, M. (2007). Improvement of coordinate measuring arm accuracy, Proceedings of the 2007 IEEE/RSJ International Conference on Intelligent Robots and Systems, 697-702, doi:10.1109/IROS.2007.4399098

[12] Vrhovec, M.; Kovac, I.; Munih, M. (2010). Measurement and compensation of deformations in coordinate measurement arm, International Symposium on Optomechatronic Technologies (ISOT), 1-6, doi:10.1109/ISOT.2010.5687319

[13] González-Madruga, D.; Cuesta, E.; Barreiro, J.; Martinez-Pellitero, S.; Alvarez, B. J. (2012). Real-time contact force measurement system for portable coordinate measuring arms, Annals of DAAAM for 2012 \& Proceedings of the $23^{\text {rd }}$ International DAAAM Symposium, Vol. 23, No. 1, 267-273

[14] González-Madruga, D.; Cuesta, E.; Barreiro, J.; Fernández-Abia, A. I. (2013). Application of a force sensor to improve the reliability of measurement with articulated arm coordinate measuring machines, Sensors, Vol. 13, No. 8, 10430-10448, doi:10.3390/s130810430

[15] Cuesta, E.; Gonzalez-Madruga, D; Alvarez, B. J.; Barreiro, J. (2014). A new concept of featurebased gauge for coordinate measuring arm evaluation, Measurement Science and Technology, Vol. 25, No. 6, paper 065004, doi:10.1088/0957-0233/25/6/065004

[16] ASME B89.4.22 (2004). Methods for performance evaluation of articulated arm coordinate measuring machines, American Society of Mechanical Engineers, New York

[17] VDI/VDE 2617 (2009). Part 9 - Acceptance and reverification test for articulated arm coordinate measuring machines, Part 7 - Accuracy of coordinate measuring machines parameters and their checking. Estimation of measurement uncertainty of coordinate measuring machines by means of simulation, Verein Deutscher Ingenieure, Düsseldorf

[18] ISO/DIS 10360-12 (2014). Geometrical Product Specifications (GPS) - Acceptance and reverification tests for coordinate measuring systems (CMS) - Part 12: Articulated arm coordinate measurement machines (CMM), Int. Organization for Standardization, Geneva

[19] Santolaria, J.; Brau, A.; Velazquez, J.; Aguilar, J. J. (2010). A self-centering active probing technique for kinematic parameter identification and verification of articulated arm coordinate measuring machines, Measurement Science and Technology, Vol. 21, No. 5, paper 055101, doi: 10.1088/0957-0233/21/5/055101

[20] Luque, P.; Mantaras, D. A.; Pello, A. (2013). Racing car chassis optimization using the finite element method, multi-body dynamic simulation and data acquisition, Proceedings of the Institution of Mechanical Engineers, Part P: Journal of Sports Engineering and Technology, Vol. 227, No. 1, 3-11, doi:10.1177/1754337112444517

[21] Zhao, D. X.; Yang, Y.; Xu, W.; Ding, G. L.; Peng, L. (2013). Rigid-flexible coupling-based CNC machine tool feed drive system co-simulation, Advanced Materials Research, Vols. 694-697, 115-119, doi:10.4028/www.scientific.net/AMR.694-697.115

[22] Cirkovic, B.; Camagic, I.; Vasic, N.; Burzic, Z.; Folic, B. (2015). Analysis of the supporting structure of composite material tool machine using the finite element method, Technical Gazette, Vol. 22, No. 1, 95-98, doi:10.17559/TV-20130928105016

[23] Mahmood, A.; Wang, X.-W., Zhou, C.-W. (2011). Modeling strategies of 3D woven composites: A review, Composite Structures, Vol. 93, No. 8, 1947-1963, doi:10.1016/j.compstruct.2011. $\underline{03.010}$

[24] Zhao, Q.; Hoa, S. V. (2003). Triaxial woven fabric (TWF) composites with open holes (Part I): Finite element models for analysis, Journal of Composite Materials, Vol. 37, No. 9, 763-789, doi: $10.1177 / 002199803031054$

[25] Bowman, C. L.; Roberts, G. D.; Braley, M. S.; Xie, M.; Booker, M. (2003). Mechanical properties of triaxial braided carbon/epoxy composites, $35^{\text {th }}$ International SAMPE Technical Conference, Dayton 